\title{
Difference in seroprevalence of IgG antibodies against hepatitis $A$ virus on general population in 1998 and 2019 in Iwate Prefecture, Japan
}

\author{
Akio Miyasaka ( $\nabla$ akimiya@iwate-med.ac.jp ) \\ Iwate Medical University https://orcid.org/0000-0001-7747-5977 \\ Yuichi Yoshida \\ Iwate Medical University \\ Akiko Suzuki \\ Iwate Medical University \\ Yasuhiro Takikawa \\ Iwate Medical University
}

\section{Short report}

Keywords: Hepatitis A virus, IgG antibodies, Prevalence, Immunity, Population

Posted Date: April 28th, 2020

DOI: https://doi.org/10.21203/rs.3.rs-24658/v1

License: (c) (i) This work is licensed under a Creative Commons Attribution 4.0 International License.

Read Full License 


\section{Abstract}

Background: Hepatitis A virus (HAV) infection, a sporadically occurring disease in general populations, is a major cause of acute hepatitis, but rarely causes death. It is important to know the current situation regarding this infection rate among the general population so that an effective strategy and action plan for HAV vaccination or treatment can be established. Here, we investigated the seroprevalence of IgG antibodies against HAV (anti-HAV) in Iwate Prefecture, Japan, in 1998 and 2019, and compared the seroprevalence patterns over these past two decades.

Methods: Altogether, 777 randomly selected serum samples were obtained from health check-up residents in Iwate prefecture in 1998 and 2019. Serum anti-HAV IgG levels were measured by radioimmunoassay (in 1998) and chemiluminescent immunoassay (in 2019).

Results: Altogether, 586 serum samples ( 278 from males, 308 from females; mean age 53 years; range 30-89 years) and 191 serum samples ( 96 from males, 95 from females; mean age 54 years; range 3089 years) from 1998 and 2019 were tested, respectively. The overall anti-HAV IgG prevalence in 2019 [16.8\% (95\% confidence interval, $\mathrm{Cl} ; 1.8-22.8 \%)]$ was significantly lower than that in $1998[47.8 \%(95 \% \mathrm{Cl}$ $43.7-51.9 \%)](p<0.001)$. Subgroup analysis of each age group showed that the anti-HAV IgG prevalence tended to be low in the young, increased with age, and each age group's prevalence became older across the two decades. The anti-HAV IgG prevalence for those older than 50 years in 2019 [28.1\% (95\% Cl 11.8$22.8 \%)]$ was significantly lower than that in $1998[75.6 \%(95 \% \mathrm{Cl} 43.7-51.9 \%)](\mathrm{p}<0.001)$.

Conclusions: A shift toward older across in anti-HA IgG prevalence was observed from 1998 to 2019, revealing that the younger population lacks immunity to HAV. Thus, selective HAV vaccination might be necessary for prevent severe HAV infection.

\section{Introduction}

Hepatitis A virus (HAV) is a single-stranded RNA virus belonging to the Picornavirus family [1]. Its particles were first detected in stool specimens from patients with acute hepatitis in 1973 by immune electron microscopy [2]. HAV is a major cause of acute hepatitis, but less than $1 \%$ of those with it progress to fulminant hepatitis [3]. Although the route of infection among men who have sex with men has been reported [4], HAV is mainly transmitted via polluted water and contaminated foods, or through the fecal-oral route. Improved hygiene and sanitary conditions have reduced the incidence of hepatitis A infections, but outbreaks still occur sporadically.

Protective antibodies against HAV (anti-HAV) are inducible by infection or vaccination. Both anti-HAV IgM and IgG are detectable during the early stage of an acute hepatitis A infection. Although IgM anti-HAV levels usually diminish within 2 to 3 months post-infection, anti-HAV IgG confers life-long immunity, and individuals who lack anti-HAV IgG can become infected with HAV. Therefore, it is important to know the current situation of HAV infection among the general population so that an effective strategy and action plan for HAV vaccination or treatment can be established. 
In the present study, we aimed to determine the seroprevalence of anti-HAV IgG in Iwate Prefecture in 1998 and 2019 to enable us to assess whether HAV seroprevalence has changed over the past two decades. Our data will be useful for determining the most appropriate way forward to safeguard people against HAV infection.

\section{Materials And Methods}

\section{Serum samples}

Assuming an expected $2 \%$ positivity rate for the group and $2 \%$ absolute accuracy, the sample size was calculated as $1.96^{2} \times(0.02) \times(1-0.02) /(0.02)^{2}=188.2$.

Altogether, 777 serum samples were randomly selected and obtained from health check-up residents in Iwate prefecture in 1998 and 2019. All of these were from residents aged 30-89 years. Samples were collected at association for preventive medicine of Iwate, and immediately stored at $-20^{\circ} \mathrm{C}$ until testing.

The study was carried out according to the Declaration of Helsinki and approved by the Ethics Committee of Iwate Medical University (approval number MH2019-078). Informed consent was obtained verbally and/or opt-out document.

\section{Anti-HAV IgG measurements}

According to the manufacturers' instructions, the serum anti-HAV IgG levels in the samples from 2019 were measured by chemiluminescent immunoassay (CLIA; SRL Inc., Tokyo, Japan), and by anti-HAV IgG Radioimmunoassay (RIA; SRL Inc., Tokyo, Japan) in 1998. The cutoff values for CLIA and RIA were 1.0 (signal/cutoff) and 1.5 (C-index), respectively.

The overall HAV prevalence was estimated based on the number of anti-HAV IgG positive samples among the total number of serum samples analyzed. Thereafter, the samples were divided into the following age groups based on 5-year intervals: $30-34,35-39,40-44,45-49,50-54,55-59,60-64,65-69,70-74$, $75-79,80-84$, and $85-89$. Subgroup analysis was performed on each age group.

\section{Statistical analysis}

A 95\% confidence interval $(95 \% \mathrm{Cl})$ was calculated for each prevalence rate. Categorical variables were analyzed using Chi-square and Fisher's exact tests. P-values of $<0.05$ (determined by a two-tailed test) were considered statistically significant. All statistical analyses were conducted using SPSS software (SPSS Statistics for Windows, SPSS Inc., IL, USA).

\section{Results}

\section{Anti-HAV IgG prevalence rates in 1998 and 2019}


Altogether, 586 serum samples ( 278 from males, 308 from females, mean age 53 years, range $30-$ 89 years) and 191 serum samples (96 from males, 95 from females, mean age 54 years, range 3089 years) were collected during 1998 and 2019, respectively.

In 1998, 280 serum samples were tested for anti-HAV IgG positivity, and the overall anti-HAV IgG prevalence was $47.8 \%$ (95\% Cl 43.7-51.9\%). In contrast, in 2019, 32 serum samples were tested for antiHAV IgG positivity, and the overall anti-HAV IgG prevalence was $16.8 \%$ (95\% Cl 1.8-22.8\%). Therefore, the overall anti-HAV IgG prevalence in 2019 was much lower than in $1998(p<0.001)$.

\section{Anti-HAV IgG prevalence in each age group in 1998 and 2019}

We calculated anti-HAV IgG prevalence in each age group as a subgroup analysis for 1998 and 2019 (Table).

Table. Anti-HAV IgG prevalence in 1998 and 2019 by age group

\begin{tabular}{|c|c|c|c|c|c|c|}
\hline \multirow{3}{*}{$\begin{array}{l}\text { Age (years) } \\
30-34\end{array}$} & \multicolumn{3}{|l|}{1998} & \multicolumn{3}{|l|}{2019} \\
\hline & \multicolumn{2}{|c|}{ Anti-HAV prevalence } & \multirow{2}{*}{$\begin{array}{l}95 \% \mathrm{Cl} \\
0-15.3\end{array}$} & \multicolumn{2}{|c|}{ Anti-HAV prevalence } & \multirow{2}{*}{$\begin{array}{l}95 \% \mathrm{Cl} \\
0-23.8\end{array}$} \\
\hline & $0 \%$ & $(0 / 18)$ & & $0 \%$ & $(0 / 11)$ & \\
\hline $35-39$ & $4.9 \%$ & $(4 / 81)$ & $1.4-12.2$ & $0 \%$ & $(0 / 29)$ & $0-9.8$ \\
\hline $40-44$ & $5.3 \%$ & $(4 / 76)$ & $1.5-12.9$ & $0 \%$ & $(0 / 20)$ & $0-13.9$ \\
\hline $45-49$ & $30.6 \%$ & $(26 / 85)$ & $21.0-41.5$ & $0 \%$ & $(0 / 19)$ & $0-14.6$ \\
\hline $50-54$ & $54.4 \%$ & $(56 / 103)$ & $44.3-64.2$ & $0 \%$ & $(0 / 22)$ & $0-12.7$ \\
\hline $55-59$ & $67.8 \%$ & $(40 / 59)$ & $54.4-79.4$ & $5.6 \%$ & $(1 / 18)$ & $0.1-27.3$ \\
\hline $60-64$ & $83.3 \%$ & $(45 / 54)$ & $70.7-92.1$ & $14.3 \%$ & $(4 / 28)$ & $4.0-32.7$ \\
\hline $65-69$ & $92.6 \%$ & $(50 / 54)$ & $82.1-97.9$ & $16.7 \%$ & $(2 / 12)$ & $2.1-48.4$ \\
\hline $70-74$ & $96.2 \%$ & $(25 / 26)$ & $80.4-99.9$ & $60.0 \%$ & $(6 / 10)$ & $26.2-87.8$ \\
\hline $75-79$ & $100 \%$ & $(20 / 20)$ & $86.1-100$ & $80.0 \%$ & $(8 / 10)$ & $44.4-97.5$ \\
\hline $80-84$ & $100 \%$ & $(7 / 7)$ & $65.2-100$ & $87.5 \%$ & $(7 / 8)$ & $47.3-99.7$ \\
\hline $85-89$ & $100 \%$ & $(3 / 3)$ & $36.8-100$ & $100 \%$ & $(4 / 4)$ & $47.3-100$ \\
\hline Total & $47.8 \%$ & $(280 / 586)$ & $43.7-51.9$ & $16.8 \%$ & $(32 / 191)$ & $1.8-22.8$ \\
\hline
\end{tabular}

Anti-HAV IgG prevalence tended to be low in younger age groups, increasing with age, as was observed in both sampling years (1998 and 2019). Although anti-HAV IgG antibodies in 1998 were rarely detected in 
individuals aged between 30-34 years, seroprevalence gradually increased from 35-39 years of age onwards. However, anti-HAV IgG prevalence among individuals aged between $30-54$ years was very low in 2019, and seroprevalence gradually increased in those aged 55-59 years. Anti-HAV IgG prevalences in those aged older than 50 years were $75.6 \%$ (95\% Cl 43.7-51.9\%) in 1998 and $28.1 \%$ (95\% Cl 11.8-22.8\%) in 2019. The anti-HAV IgG prevalence in individual aged older than 50 years in 2019 was much lower than that in $1998(p<0.001)$.

The changes in anti-HAV IgG prevalence in each age group during the past two decades is depicted in Fig. 1. The shapes of the prevalence curves are sigmoidal, with age-related anti-HAV IgG positivity shifting rightwards over the 20-year period.

\section{Discussion}

Nationwide anti-HAV IgG seroprevalence studies were conducted among the general population of Japan in 1973, 1984, 1994, and 2003 [5-7]. By combining the data from these reports, the overall anti-HAV IgG prevalence in 1994 was $19.4 \%$ and $12.2 \%$ in 2003 . Shifting toward older across anti-HAV IgG prevalence in each age group was also observed. Anti-HAV IgG prevalence tended to be low in younger people but increased yearly with age. Anti-HAV IgG prevalence in individuals older than 50 years was $96.9 \%$ in 1973 , $96.9 \%$ in $1984,74.3 \%$ in 1994 , and $50.3 \%$ in 2003 . Therefore, the overall anti-HAV IgG prevalence in the population and that for the over 50 s decreased gradually each year. One possible explanation for this is improved hygiene and sanitary conditions in the population. After 2003, reports on anti-HAV IgG prevalence among the general population were not documented, except for in 2015 where seroprevalence among the general population of Hiroshima Prefecture was reported [8]. This report mentioned that the overall anti-HAV IgG prevalence rate was $16.8 \%$, while that of anti-HAV IgG in individuals aged over 50 years was $33.2 \%[8]$.

According to the Infectious Agent Surveillances Reports (https://www.niid.go.jp/niid/ja/) from April 1999 to March 2019 and the HAV incidence reports [9, 10], sporadic HAV outbreaks occurred nationwide in 1999, 2010, 2014 and 2018. Our hospital survey showed that the number of inpatients with acute hepatitis A infections in our hospital from 1998 to 2019 in each year was low, except for the 1998 epidemic (Fig. 2). The present seroprevalence study from Iwate Prefecture among the general population during 1998 and 2019 showed that the overall anti-HAV IgG prevalence in 2019 was significantly lower than that in 1998. Anti-HAV IgG prevalence rates in each age group also shifted towards older people during the two decades. Our hospital survey findings, the seroprevalence age shift, and the low prevalence seen in the present study suggest a low population transmission rate and low viral infectivity.

Those at highest risk of contracting HAV infections are the young because they have low immunity to this infection. Japanese healthcare workers are also reported to be at risk of occupational HAV infection because their immunity to HAV will have decreased with age [11]. Older people without anti-HAV IgGrelated immunity may contract a more serious infection [12]. Furthermore, international travel to Japan can also bring foreigners from HAV endemic areas to the country; likewise for Japanese people who have 
travelled to areas where HAV is endemic. Although personal hygiene and sanitary environmental conditions are important, an HAV vaccine is available that offers long-term protection against HAV infection. One drawback is that HAV vaccination is necessary twice every two weeks to achieve protective antibody levels, but three doses are needed to maintain long-term protective antibody levels [13]. Currently, voluntary HAV vaccination is administered from childhood to adulthood in Japan. The vaccination, although voluntary, is strongly recommended for those with a higher risk of infection with HAV than others, such as travelers to HAV endemic areas, medical practitioners with a high chance of contact with HAV patients, and men who have sex with men. The available HAV vaccine might be introduced to the general population, particularly the young generation.

This study has several limitations. First, the measurement method for HAV serodiagnosis has changed over the last 20 years, so the measurement method used for the 1998 and 2019 samples differs in the present study. However, the overall trend seems not to have changed. Second, because the serum samples were provided by people during their medical checkups, the samples for those aged $0-30$ years have not been tested. Moreover, there are few samples from the elderly. Third, because we tested the samples randomly, possible differences in anti-HAV IgG prevalence between males and females could not be compared. However, recent studies report that there is no difference in anti-HAV IgG prevalence between males and females [6,7].

To conclude, population surveillance of anti-HAV IgG prevalence is useful for designing preventive measures against the spread of HAV. Our seroprevalence study for 1998 and 2019 in Iwate Prefecture showed that the anti-HAV IgG prevalence in each age group had shifted towards older people over two decades, and that younger people lack immunity to HAV. Selective use of HAV vaccination warrants consideration.

\section{Abbreviations}

Anti-HAV: anti-hepatitis A virus antibody; Chemiluminescent immunoassay (CLIA); HAV: Hepatitis A virus; Radioimmunoassay: RIA; 95\% Cl: 95\% confidence interval

\section{Declarations}

\section{Ethics approval and consent to participate}

All procedures followed were performed in accordance with the ethical standards laid down in the 1964 Declaration of Helsinki and its later amendments. The Ethics Committee of Iwate Medical University approved this study (approval number MH2019-078). Informed consent was obtained verbally and/or opt-out document.

\section{Consent for publication}

No individually identifiable pattern information or images are present in this report. 


\section{Competing interests}

The authors declare that they have no competing interests.

\section{Funding}

This study was partly supported by a Grant from the Ministry of Health, Labor and Welfare, Japan.

\section{Authors' contributions}

AM conceived and designed the study. AM, YY, and AS analyzed the data. YT supervised this work. AM wrote the manuscript. The authors have read and approved the final manuscript.

\section{Acknowledgments}

We thank Association for Preventive Medicine of Iwate and the residents of the prefecture for providing samples. We also thank Sandra Cheesman, PhD, from Edanz Group (www.edanzediting.com/ac) for editing a draft of this manuscript.

\section{References}

1. Lemon SM, Ott JJ, Van Damme P, Shouval D. Type A viral hepatitis: A summary and update on the molecular virology, epidemiology, pathogenesis and prevention. J Hepatol. 2017;68:167-84.

2. Feinstone SM, Purcell RH. Hepatitis A: detection by immune electron microscopy of a viruslike antigen associated with acute illness. Science. 1973;182:1026-8.

3. Rezende G, Roque-Afonso AM, Samuel D, Gigou M, Nicand E, Ferre V, et al. Viral and clinical factors associated with the fulminant course of hepatitis A infection. Hepatology. 2003;38:613-8.

4. Corey L, Holmes KK. Sexual transmission of hepatitis A in homosexual men: incidence and mechanism. N Engl J Med. 1980;302:435-8.

5. Taylor-Wiedeman J, Moritsugu Y, Miyamura K, Yamazaki S. Seroepidemiology of hepatitis A virus in Japan. Jpn J Med Sci Biol. 1987;40:119-30.

6. Kiyohara T, Sato T, Totsuka A, Miyamura T, Ito T, Yoneyama T. Shifting seroepidemiology of hepatitis A in Japan, 1973-2003. Microbiol Immunol. 2001;51:185-91.

7. Kiyohara T, Satoh T, Yamamoto H, Totsuka A, Moritsugu Y. The latest seroepidemiological pattern of hepatitis A in Japan. Jpn J Med Sci Biol. 1997;50:123-31.

8. Yamamoto C, Ko K, Nagashima S, Harakawa T, Fujii T, Ohisa M, et al. Very low prevalence of anti-HAV in Japan: high potential for future outbreak. Sci Rep. 2019;9:1493.

9. Ishii K, Kiyohara T, Yoshizaki S, Wakita T, Shimada T, Nakamura N, et al. Epidemiological and genetic analysis of a diffuse outbreak of hepatitis A in Japan 2010. J Clin Virol. 2012;53:219-24. 
10. Ishii K, Kiyohara T, Yoshizaki S, Kawabata K, Kanayama A, Yahata Y, et al. Epidemiological and genetic analysis of a 2014 outbreak of hepatitis A in Japan. Vaccine. 2015;33:6029-36.

11. Yoshioka N, Deguchi M, Hagiya H, Kagita M, Tomono K. Reduced seroprevalence of hepatitis A virus among Japanese healthcare workers as a risk factor for occupational infection. Clin Lab. 2018;64:1791-3.

12. Nakao M, Nakayama N, Uchida $\mathrm{Y}$, Tomiya T, Oketani M, Ido A, et al. Deteriorated outcome of recent patients with acute liver failure and late-onset hepatic failure caused by infection with hepatitis $A$ virus: A subanalysis of patients seen between 1998 and 2015 and enrolled in nationwide surveys in Japan. Hepatol Res. 2019;49:844-52.

13. Fukushima S, Kiyohara T, Ishii K, Nakano T, Hamada A. Immunogenicity of aluminum-adsorbed hepatitis A vaccine (Havrix $\AA$ ) administered as a third dose after primary doses of Japanese aluminum-free hepatitis A vaccine (Aimmugen ${ }^{\circledR}$ ) for Japanese travelers to endemic countries. Vaccine. 2017;35:6412-5.

\section{Figures}

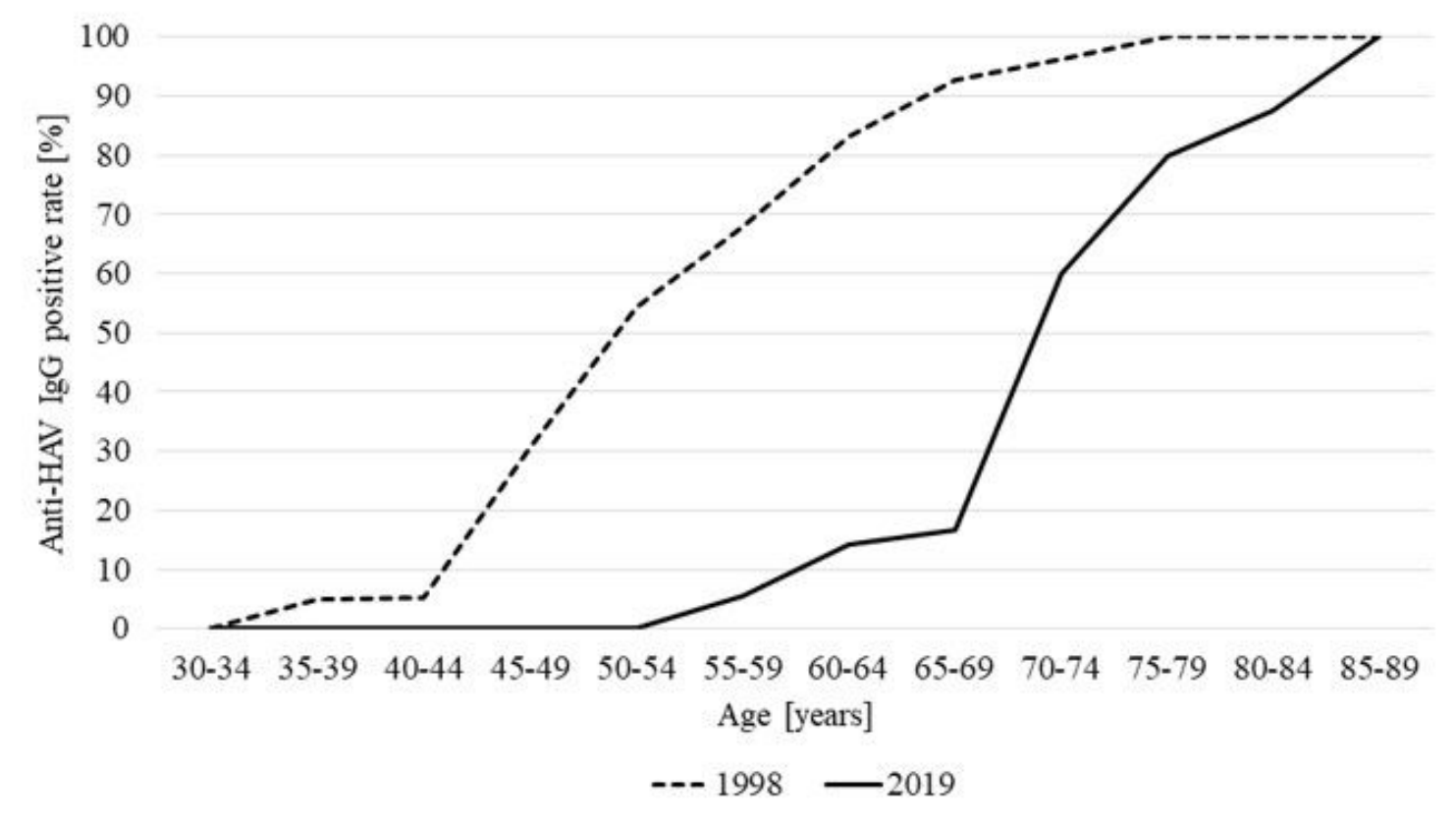

\section{Figure 1}

Age-specific seroprevalence of anti-HAV IgG during 1998 and 2019 Anti-HAV IgG seroprevalence among the general population of Iwate Prefecture, Japan, in 1998 (dotted line) and 2019 (solid line). The seroprevalence for each age group has shifted rightwards in the graph over the two decades. 


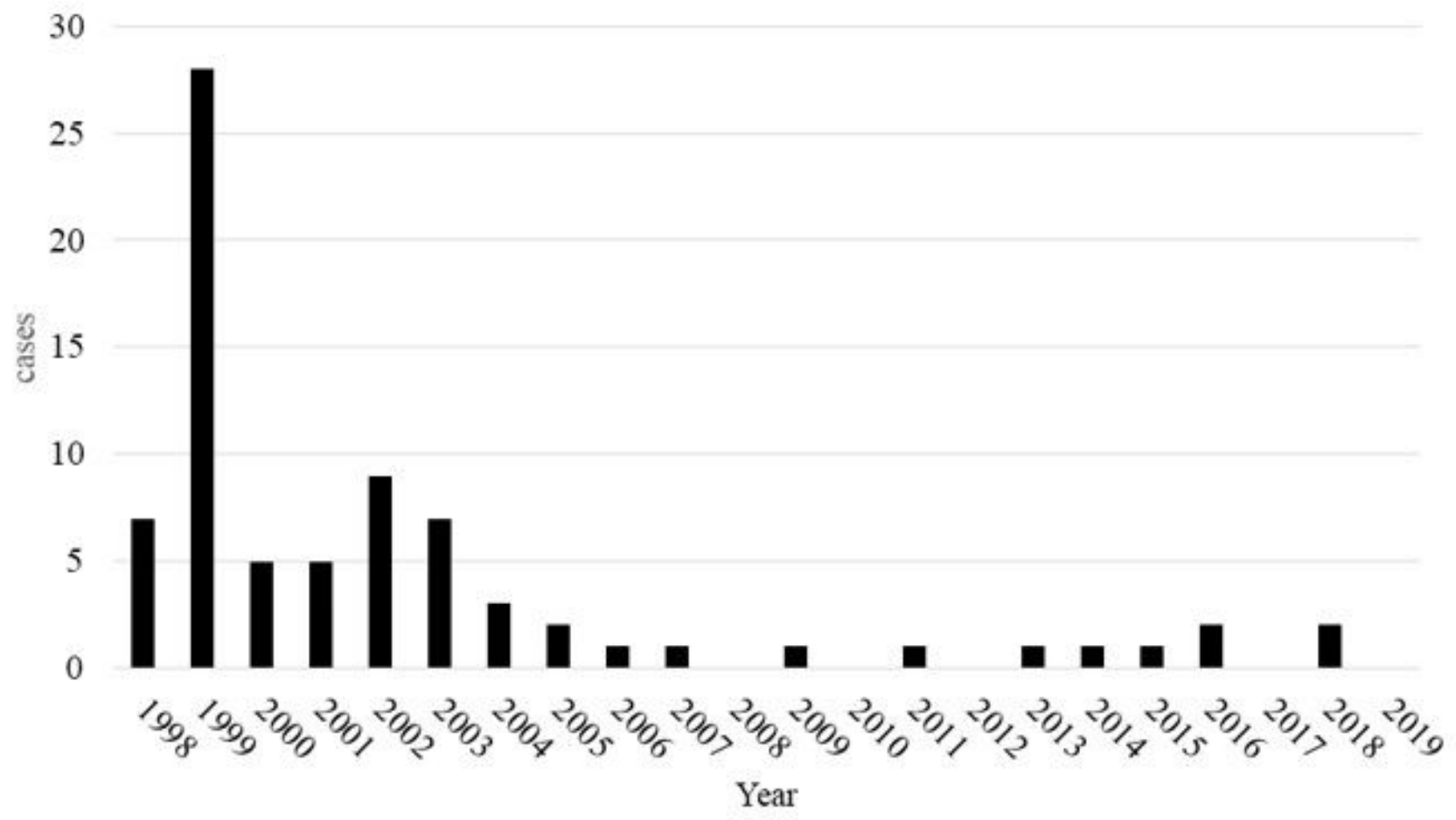

Figure 2

Incidence of acute hepatitis A in our hospital during 1998-2019 Graph for the yearly number of inpatients with acute hepatitis A in our hospital from 1998 to 2019. HAV occurrence in each year was low except for in 1998, the epidemic year. 